\title{
Effect of variation sintering temperature on magnetic permeability and grain sizes of Y3Fe5012 via mechanical alloying technique
}

\begin{abstract}
his work will focus on the preparation of yttrium iron garnet (Y3Fe5O12, YIG) via mechanical alloying technique derive by steel waste product. The $\mathrm{Fe} 2 \mathrm{O} 3$ powder derived from the steel waste purified by using magnetic and non-magnetic particles (MNM) and Curie temperature separation (CTS) technique. The purified powder was then oxidized in air at $500{ }^{\circ} \mathrm{C}$ for 9 hours in air. The $\mathrm{Fe} 2 \mathrm{O} 3$ was mixed with $\mathrm{Y} 2 \mathrm{O} 3$ using high energy ball milling for 9 hours. The mixed powder obtained was pressed and sintered at different temperature $500 / 600 / 700 / 800 / 900 / 1000 / 1100{ }^{\circ} \mathrm{C}$. X-ray diffraction (XRD) shows the YIG is completely form at $1100{ }^{\circ} \mathrm{C}$. The field emission scanning electron microscopy (FESEM) images shows the grain size increases as increase the sintering temperatures. The frequency dependence on the complex permeability, $\mu$ ' and magnetic loss, $\mu$ ' in the frequency range $10 \mathrm{MHz}$ to $1 \mathrm{GHz}$ were measured in this study. The results showed that the highest $\mu^{\prime}$ is 5.890 obtained from $1100{ }^{\circ} \mathrm{C}$.
\end{abstract}

Keyword: Porous cavity; Wavy wall; Thermal dispersion; Nanofluid; Numerical results 
